\title{
Revisiting astronomical crystalline forsterite in the UV to near-IR
}

\author{
Karly M. Pitman ${ }^{1}$, Anne M. Hofmeister ${ }^{2}$, and Angela K. Speck ${ }^{3}$ \\ ${ }^{1}$ Planetary Science Institute, 1700 East Fort Lowell Road, Suite 106, Tucson, AZ 85719-2395, USA \\ ${ }^{2}$ Department of Earth \& Planetary Sciences, Washington University, Campus Box 1169, 1 Brookings Drive, St. Louis, MO 63130-4899, USA \\ ${ }^{3}$ Department of Physics \& Astronomy, 223 Physics Bldg., University of Missouri, Columbia, MO 65211-7010, USA
}

(Received December 31, 2011; Revised May 8, 2012; Accepted May 11, 2012; Online published March 12, 2013)

\begin{abstract}
Optical functions ( $n$ and $k$ ) of cosmic dust species like forsterite $\left(\mathrm{Mg}_{2} \mathrm{SiO}_{4}\right)$ are required at all wavelengths to quantify the temperature and amount of dust. Astronomers combine optical functions of forsterite and olivine in different ways, which will affect radiative transfer models. We investigated what recent updates to the ultraviolet-visible-near-infrared (UV-VIS-NIR) laboratory spectra of forsterite and the choice of forsterite $n, k$ dataset will have on radiative transfer models. We measured the UV-VIS-NIR transmission spectra of synthetic forsterite, $\mathrm{MgO}, \mathrm{SiO}_{2}$, olivine ( $\mathrm{Fo} 90$ ), and meteoritic olivine (pallasite). We derived optical functions for these and compared the UV-IR behavior of our $k$, absorption cross-section $\left\langle C_{\mathrm{abs}}\right\rangle$, and total flux to that of "astronomical silicate" and olivine. Laboratory-derived $k$ is substantially lower than "astronomical silicate" $k$ at $\lambda \sim 0.2-5 \mu \mathrm{m}$. In the IR, different laboratory $n$ and $k$ produce equivocal $\left\langle C_{\text {abs }}\right\rangle$, whereas total flux is different for "astronomical silicate" versus laboratory $n, k$. From $0.35-5 \mu \mathrm{m}$, the choice of "forsterite" $k$ values has the most effect on modeled quantities. For environments with significant UV flux, astronomers should use recent UV-VIS-NIR laboratory $n, k$.
\end{abstract}

Key words: Dust, extinction, forsterite, optical constants, ultraviolet, near-infrared, laboratory spectroscopy.

\section{Introduction}

Optical and dielectric functions of cosmic dust species are required not only to properly correct astronomical light budgets for the amount of light extinction by dust but also to understand the composition and physical nature of the dust itself in many space environments. Whereas there is a general lack of optical functions (real and imaginary indices of refraction $n$ and $k$ ) for many cosmic dust species, there are in fact several choices of laboratory and optical function data over the electromagnetic spectrum and different temperatures for important species, e.g., crystalline and disordered olivine $\left(\mathrm{Mg}_{2 x} \mathrm{Fe}_{2-2 x} \mathrm{SiO}_{4}\right)$ and its $\mathrm{Mg}$-endmember forsterite (e.g., Huffman and Stapp, 1973; Weeks, 1974; Reynard, 1991; Jäger et al., 1994; Pollack et al., 1994; Colangeli et al., 1995; Dorschner et al., 1995; Henning et al., 1999 and references therein; Fabian et al., 2001; Suto et al., 2006; Koike et al., 2006; Sogawa et al., 2006; Pitman et al., 2010 and references therein). Modelerconstructed "cosmic," "circumstellar," or "astronomical" silicates, based in part on real mineral data, also exist (e.g., Ossenkopf et al., 1992; David and Pegourie, 1995; Draine, 2003a, b). Astronomers rely on their best judgment to combine the available laboratory or modeler-constructed optical functions and cover as many wavelengths as possible; however, for the same dust species, different groups use different assumptions in their radiative transfer analyses on how $n$ and $k$ behave across the electromagnetic spectrum. For ex-

Copyright (c) The Society of Geomagnetism and Earth, Planetary and Space Sciences (SGEPSS); The Seismological Society of Japan; The Volcanological Society of Japan; The Geodetic Society of Japan; The Japanese Society for Planetary Sciences; TERRAPUB.

doi:10.5047/eps.2012.05.009 ample, Wright et al. (2011) recently modeled the planetary nebula NGC 6302, an object that shows spectral features attributable to forsterite, using the 3D photoionization and radiative transfer Monte Carlo code MOCASSIN (Ercalano et al., 2003, 2005). To obtain optical functions out to short wavelengths, they merged $n$ and $k$ derived from polarized IR crystalline forsterite laboratory data by Sogawa et al. (2006) with the constructed UV-optical range of "astronomical silicate" $n$ and $k$ from Draine (2003a, b), which represent silicates with higher $\mathrm{Fe}^{2+}$ content and disordered (or crystalline at short wavelengths) lattice structures. By contrast, in the radiative transfer analysis of the Orion A protostar HOPS-68, Poteet et al. (2011) used a different combination of laboratory optical functions to represent forsterite: $(\mathrm{Mg}, \mathrm{Fe})_{2} \mathrm{SiO}_{4}$ from Huffman and Stapp (1973), the San Carlos olivine from Zeidler et al. (2011), $\mathrm{Mg}_{2} \mathrm{SiO}_{4}$ from Sogawa et al. (2006), and modified $\mathrm{Mg}_{1.9} \mathrm{Fe}_{0.1} \mathrm{SiO}_{4}$ values from Fabian et al. (2001) in certain wavelength regimes $(0.1-0.16,0.24-2,4-100$, and $100-8000 \mu \mathrm{m})$. If publications from the same year are in disagreement on what represents the same dust species, then which $n$ and $k$ values are the best representation of material in space? In this work, we focus on four optical function datasets used to approximate forsterite in astronomical models of dust, to provide recommendations on how to make an educated decision regarding which values are consistent and appropriate for modeling dust. We update $k$ values in the transparent, near-IR region and use previous data in the far-UV on $\mathrm{MgO}$ and $\mathrm{SiO}_{2}$ to provide complete wavelength coverage for forsterite. 


\section{Techniques}

\subsection{Laboratory spectroscopy}

We studied the spectral behavior of synthetic forsterite $\left(\mathrm{Mg}_{2} \mathrm{SiO}_{4}\right)$ and two related compounds: magnesium oxide $(\mathrm{MgO})$ and fused silica $\left(\mathrm{SiO}_{2}\right)$. Our synthetic forsterite sample was a $3.6-\mathrm{mm}$ thick single crystal created via the Verneuil flame-fusion method (Shankland and Hemmenway, 1963; Shankland, 1967), oriented along the (001) plane. Our semiconductor-grade (i.e., better than $99.9 \%$ purity) $\mathrm{MgO}$ sample was a $10-\mathrm{mm}$ cube oriented along the (100) plane, purchased from SPI Supplies / Structure Probe Inc. (West Chester, Pennsylvania, USA). The manufacturer-reported impurities constituting the $0.1 \%$ in the $\mathrm{MgO}$ sample are as follows, in maximum ppm: Ca: 40 , Al: 15, Si: 10, Fe: 50, Cr: 10, B: 5, C: 10. Our fused silica sample was a 2-mm plate of Herasil manufactured by Heraeus Quartz America, LLC, Optics Division, USA. The manufacturer description of the fused silica lists $15-26 \mathrm{ppm}$ of the cations $\mathrm{Al}$ (which is $60 \%$ ), $\mathrm{Li}, \mathrm{Na}, \mathrm{K}, \mathrm{Cr}, \mathrm{Cu}, \mathrm{Ti}$, and $\mathrm{Zr}$. There is no $\mathrm{Cl}$ but about $1000 \mathrm{ppm}(\sim 0.1 \mathrm{wt} \%)$ $\mathrm{OH}^{-1}$. The latter samples were selected for their lack of internal bubbles or inclusions and their high transparency + commercial-grade polish, permitting good transmission down to the UV. All sample thicknesses reported were measured via digital micrometer.

For each of these compounds, we acquired new room temperature laboratory spectra in the combined wavelength range 0.19-5.5 $\mu \mathrm{m}$, using two instruments. In the NIR, we used an evacuated Bomem DA3.02 Fourier transform interferometer with a $\mathrm{SiC}$ source, an $\mathrm{InSb}$ detector, a $\mathrm{CaF}_{2}$ beamsplitter, and a wire-grid polarizer for oriented singlecrystals. Approximately 2000 scans were collected per spectrum. Each NIR spectrum spanned $\sim 9000-1800 \mathrm{~cm}^{-1}$ $(\lambda \sim 1.1-5.5 \mu \mathrm{m})$ at a resolution of $2 \mathrm{~cm}^{-1}$. Unpolarized UV-VIS spectra from 52630 to $9090 \mathrm{~cm}^{-1}$ ( $\lambda \sim 0.19-1.1$ $\mu \mathrm{m})$ were collected using a double-beam Shimadzu UV1800 spectrometer with $0.001 \mu \mathrm{m}$ resolution.

\subsection{Optical function derivation}

Quantities and methods for deriving optical functions are explained in detail in Pitman et al. (2008), Hofmeister et al. (2009) and Speck et al. (2011). Briefly, using the laboratory spectra and sample thicknesses $(L)$, we calculated absorption coefficients $(A)$ for forsterite, $\mathrm{MgO}$, and fused silica via Eq. (1), accounting for specular back reflections $(R)$ in the samples.

$$
A=\frac{1}{L} *\left[-\ln \left(\frac{I_{\text {trans }}}{I_{0}}\right)+2 \ln (1-R)\right]
$$

From this we calculated imaginary index of refraction $k$ as a function of frequency $v$ for the three compounds (Eq. (2)),

$$
k=\frac{A}{4 \pi v}
$$

Shortward of our detector range, we approximated $k$ for forsterite $\left(\mathrm{Mg}_{2} \mathrm{SiO}_{4}\right)$ by combining $\mathrm{MgO}$ and $\mathrm{SiO}_{2} k$, from Palik (1998); the latter data were cross-checked against our $\mathrm{MgO}$ and fused silica lab measurements longward of 0.19 $\mu \mathrm{m}$. To do the approximation, we created two columns of $k$ : one for $\mathrm{MgO}$ and one for $\mathrm{SiO}_{2}$. Then we interpolated the $\mathrm{MgO}$ and $\mathrm{SiO}_{2} k$ with a weighted average: $(2 \mathrm{MgO}+$ $\left.1 \mathrm{SiO}_{2}\right) / 3$ to get $\mathrm{Mg}_{2} \mathrm{SiO}_{4} k$. In this way, it is possible to obtain the spectral properties of not only $\mathrm{Mg}_{2} \mathrm{SiO}_{4}$ but also other magnesium silicates by adjusting the weighted average to match the stoichiometry. For $\lambda=0.19-0.36$ and 3.3$5.5 \mu \mathrm{m}$, we derived $k$ directly from our synthetic forsterite lab measurements via Eqs. (1)-(2). Because $k$ derived from our forsterite lab spectra at 0.36 to $1.10 \mu \mathrm{m}$ equals zero within experimental uncertainty, we interpolated $k$ derived from our lab measurements in the UV at $0.36 \mu \mathrm{m}$, where absorption is finite and measurable, to the near IR where weak overtones exist, at $3.3 \mu \mathrm{m}$. This further constrained our $A$ and thus $k$ from 3.3 to 5.5 microns. At 5.5-6 $\mu \mathrm{m}$, we interpolated between the end of our near-IR detector range and the beginning of literature data. At wavelengths $>6$ $\mu \mathrm{m}$, we derived $k$ from Suto et al. (2006) and Sogawa et al. (2006), which are high resolution and high signal-to-noise spectra of large, oriented samples with good performance over the $1200-100 \mathrm{~cm}^{-1}(8.3-100 \mu \mathrm{m})$ range.

To determine $n$ for forsterite, for $\lambda<0.215 \mu \mathrm{m}$, we approximated $n$ from the Palik (1998) data for $\mathrm{MgO}$ and $\mathrm{SiO}_{2}$ as above, in proportion to the stoichiometry. Between 0.215 and $0.6 \mu \mathrm{m}$, we interpolated $n$ values. We obtained $n$ in the transparent region via Eq. (3),

$$
R=\left(\frac{n-1}{n+1}\right)^{2}
$$

Typically, $n$ is measured directly in the visible and measurements of $R$ in the IR are extrapolated to the visible tiepoint $\left(n_{\mathrm{vis}}=1.65\right.$ at $\lambda=0.6 \mu \mathrm{m}$, averaged for the 3 polarizations of forsterite; Deer et al., 1992). This approach avoids problems with back reflections in the transparent region. Using $n_{\text {vis }}=1.65$, we performed Lorentz classical dispersion analysis on laboratory $R$ values from Suto et al. (2006) which constrains $n$ from the far-IR to $0.6 \mu \mathrm{m}$. The rise in $n$ is very gradual (see curves for other dust species in Palik, 1998).

\section{Results}

\subsection{Laboratory optical functions}

Figure 1 presents the real and imaginary indices of refraction $n$ and $k$ that we determined using laboratory spectra for the three orientations of forsterite: electric field vector $\vec{E}$ parallel to crystal axes $\vec{a}$ (solid line), $\vec{b}$ (dotted line), and $\vec{c}$ (dashed line). Constructed $n$ and $k$ for "astronomical silicate" by Draine (2003b), though not the same Fe content or lattice structure as the lab samples, are plotted for comparison (dash-dot line) to stress their comparison in usage by modelers (e.g., Wright et al., 2011). The ordinary ray is $\vec{E} \| \vec{a}$, and the extraordinary ray is $\vec{E} \| \vec{c}$. Wavelengths from $\lambda=0-8 \mu \mathrm{m}$ are shown, as this is the region that is most debatable when merging datasets. Electronic data tables and supplementary text files describing how the $n$ and $k$ in Figs. 1-2 were constructed and merged in this work are available at our website (see Acknowledgments for URL). Our $n$ and $k$ data are not polarized in the UV, but are polarized in the NIR and longward (generally $\lambda>3 \mu \mathrm{m}$ ).

For much of the VIS-NIR, the forsterite $n$ values that we derive are slightly lower than what Draine (2003b) finds. 

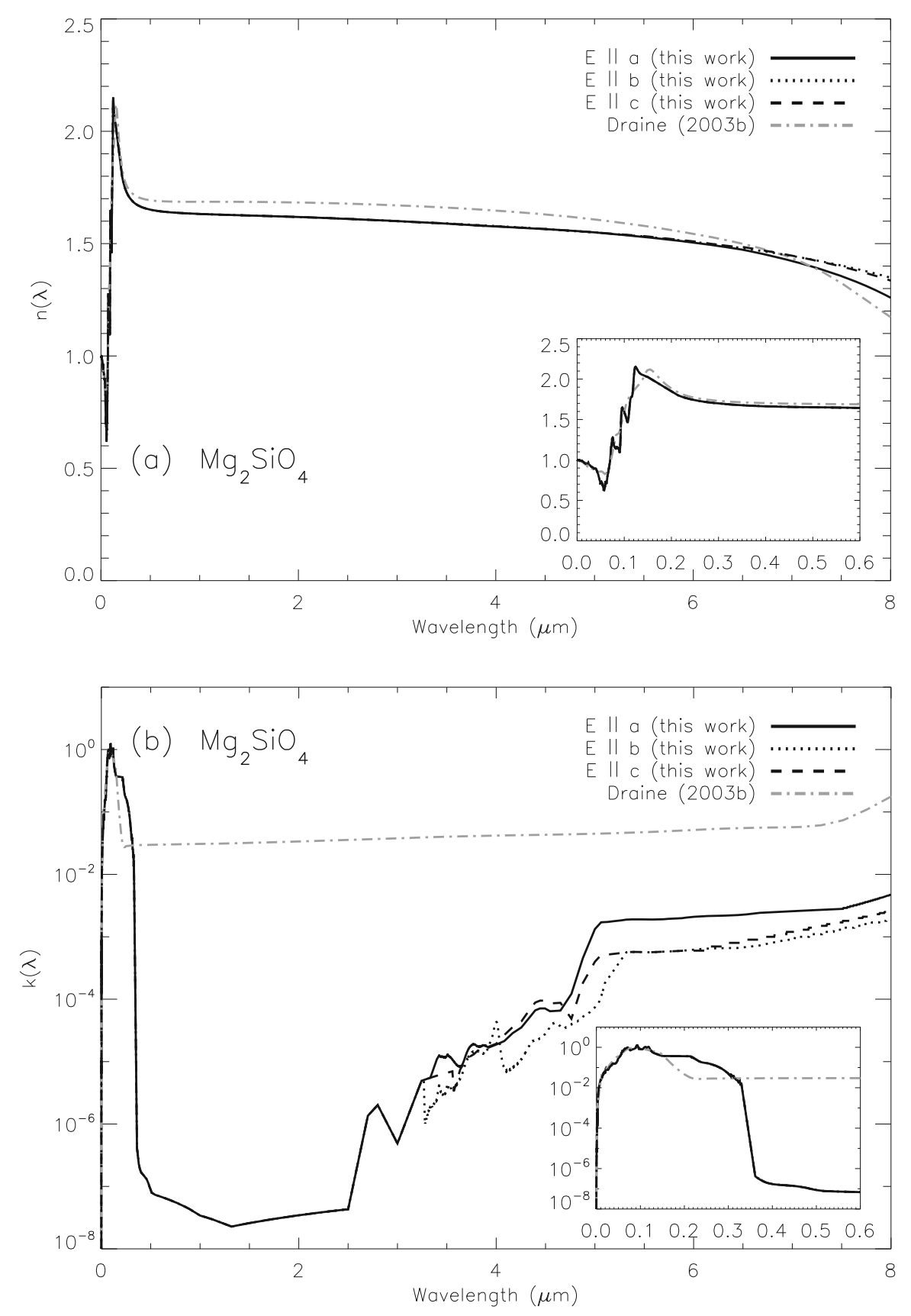

Fig. 1. Real and imaginary indices of refraction $n(\lambda)$ and $k(\lambda)$ for crystalline forsterite $\mathrm{Mg}_{2} \mathrm{SiO}_{4}$ (solid line, dotted line, dashed line). Sharp peaks in $n$ below $0.15 \mu \mathrm{m}$ are X-ray absorption peaks (panel a inset). New laboratory data segments in the mid-UV to VIS from $0.214-0.330 \mu \mathrm{m}$ and in the NIR from 3.278-5.1 $\mu \mathrm{m}$ set $k$ lower than predicted from modeler-constructed "astronomical silicate" values (Draine, 2003b; gray dash-dot line).

The largest difference is seen in $k$. Sharp peaks in $n$ below $0.15 \mu \mathrm{m}$ are the $\mathrm{K}$ and $\mathrm{L} \mathrm{X}$-ray absorption peaks of $\mathrm{O}, \mathrm{Si}$, and $\mathrm{Mg}$. Starting at approximately $0.20 \mu \mathrm{m}$, the largest peak in $k$ is more rounded and does not drop off as quickly in the laboratory data as in the constructed "astronomical silicate." This is followed by a precipitous drop in $k$, which occurs in spectral data for many materials, e.g., corundum $\left(\mathrm{Al}_{2} \mathrm{O}_{3}\right)$, for which $k$ plummets five orders of magnitude between 0.14 and $0.15 \mu \mathrm{m}$ and is below $10^{-5}$ from $\lambda=0.2-3 \mu \mathrm{m}$ (Tropf and Thomas, 1998). This steep drop in $k$ is a hallmark of crystals and glasses without coloring agents such as $\mathrm{Fe}^{2+}$. We only present $n$ and $k$ for wavelengths where $A$ is clearly greater than zero. For the transparent regions, where the corrected absorbance equals zero within experimental uncertainty, the maximum value of $A$ can be set either from spectral noise or from very weak peaks. This in turn sets constraints on $A$ at certain frequencies, e.g., where O-H absorptions or overtone-combination modes occur in the NIR. For the UV, it is only possible to ascertain the rise towards the charge transfer bands. Our laboratory spectra constrain the near-UV and NIR steepness of the slope of $k$ at $0.214-0.33 \mu \mathrm{m}$ and 3.3-5.1 $\mu \mathrm{m}$. Our $n$ and $k$ data also join well to IR laboratory data by Suto et al. (2006) and Sogawa et al. (2006). 

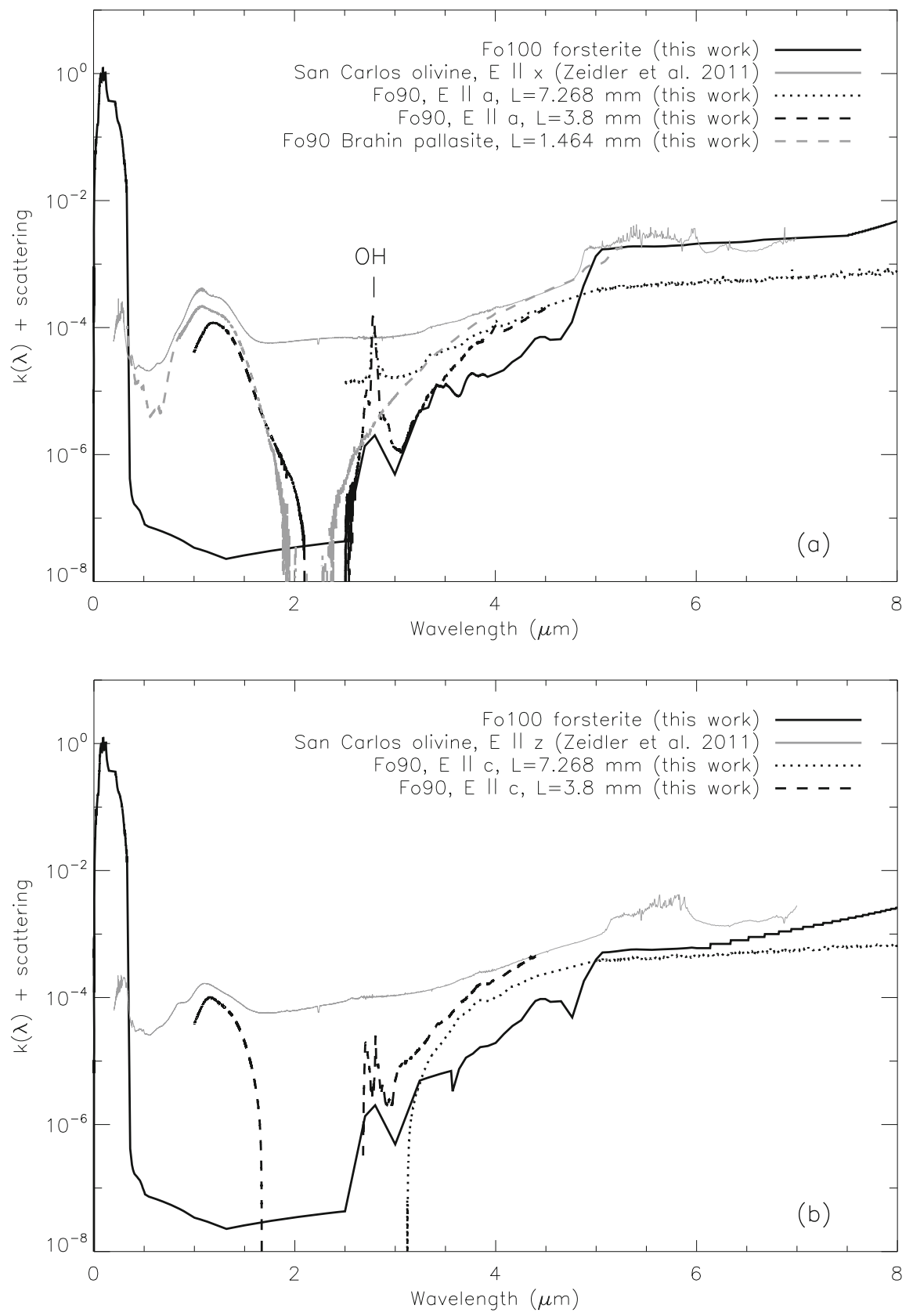

Fig. 2. Imaginary index of refraction $k(\lambda)$ for crystalline forsterite $\mathrm{Mg}_{2} \mathrm{SiO}_{4}$ from this work (solid black line), compared to olivine with $\sim 10 \%$ Fe content (gray solid line $\equiv$ San Carlos olivine from Zeidler et al., 2011; dotted and dashed lines $\equiv$ Fo90 gemstone in differing thicknesses; gray dashed line $\equiv$ Fo90 meteoritic olivine). For Fo90 $\vec{E} \| \vec{a} L=3.8 \mathrm{~mm}$ spectrum, noisy data regions due to sample thickness vs. wavelength were cut at 2.1-2.5 and 4.5-5.0 $\mu \mathrm{m}$. For Fo90 $\vec{E} \| \vec{a} L=7.268 \mathrm{~mm}$ spectrum, noisy regions were removed at 2.5-3.0 and 4.4-5.0 $\mu \mathrm{m}$. Behavior in the Fo90 data near 1 $\mu \mathrm{m}$ is at the limit of the VIS-NIR detector range and is therefore less certain.

\subsection{Comparison to past laboratory work}

There are other new laboratory data for silicates in the near-UV to NIR which must be evaluated in context with our data. For example, oriented olivine covering $0.2-6.998 \mu \mathrm{m}$ is a recent addition from Zeidler et al. (2011), who derived $k$ using table values for $n$ and transmission spectra of two varieties of olivine with different Fe content: the San Carlos and Sri Lanka samples. Our electron microprobe results indicate that the San Carlos olivine sample has a composition of $\mathrm{Mg}_{1.801} \mathrm{Fe}_{0.185} \mathrm{Mn}_{0.002} \mathrm{Ni}_{0.008} \mathrm{Ca}_{0.002} \mathrm{Si}_{1.001} \mathrm{O}_{4}$, and Downs
(2006) gives a composition for the Sri Lanka olivine sample of $\left(\mathrm{Mg}_{1.67} \mathrm{Fe}_{0.33}\right) \Sigma=2 \mathrm{Si}_{1.00} \mathrm{O}_{4}$ with trace amounts of $\mathrm{Mn}$ and $\mathrm{Ca}$, which differ from table 3 of Zeidler et al. (2011); that work gave compositions of $\mathrm{Mg}_{1.96} \mathrm{Fe}_{0.16} \mathrm{Si}_{0.89} \mathrm{O}_{4}$ for the San Carlos and $\mathrm{Mg}_{1.56} \mathrm{Fe}_{0.4} \mathrm{Si}_{0.91} \mathrm{O}_{4}$ for the Sri Lanka samples. San Carlos olivine is used as a microprobe standard due to its chemical homogeneity, and silica deficiency has not been reported. The low Si contents reported by Zeidler et al. (2011) are not consistent with literature data, suggesting problems in that chemical analysis due to standards calibration or to quality of sample polish and meaning that $\mathrm{Fe}$ 
content was not properly determined. Small samples of San Carlos olivine are uniformly Fo90.1 \pm 0.1 , whereas large stones from gem dealers have a wider range of composition (Fo89.2 \pm 0.2 to Fo91.1 \pm 0.3 ; Fournelle, 2009 and references therein). Fo90 is a reasonable estimate for the San Carlos olivine. The Zeidler et al. (2011) olivine dataset is gaining popularity as a proxy for forsterite in radiative transfer models (e.g., Poteet et al., 2011 mentioned earlier) and to our knowledge is the only recent laboratory dataset for Mg-rich silicates in a comparable wavelength range to our UV-VIS-NIR $n$ and $k$. Therefore, in Fig. 2, we directly compare our forsterite $k$ data with the San Carlos sample from Zeidler et al. (2011). (In terms of $n$, both datasets are equivalent and $n$ values are therefore not plotted.) The San Carlos sample is a better comparison than the Sri Lanka sample for this purpose because the latter is metamorphic, higher in Fe content, and contains a large amount of water. The San Carlos sample contains a little water, based on peaks near $3000 \mathrm{~cm}^{-1}(3.3 \mu \mathrm{m}$; Miller et al., 1987; Rossman, 1996; Yang and Keppler, 2011), and is slightly oxidized, which influences the UV.

Comparing the laboratory data that generated Fig. 2, we notice several things. First, at $\lambda=1 \mu \mathrm{m}$, our $\mathrm{Mg}_{2} \mathrm{SiO}_{4} k$ is approximately $3.4 \times 10^{-8}$. This is in agreement with the solid state physics literature and text in Zeidler et al. (2011), which stated that $k<8 \times 10^{-6}$ at $\lambda=1 \mu \mathrm{m}$ would be expected for transparent solids, including pure $\mathrm{MgO}, \mathrm{SiO}_{2}$, and $\mathrm{Fe}$-free $\mathrm{Mg}$-silicates. In general, the olivine $A$ values reported in Zeidler et al. (2011) are $\sim 1000$ times our forsterite $A$ in the transparent region. Zeidler et al. (2011) suggested that $k \approx 10^{-5}$ would be the lower limit at $\lambda=$ $1 \mu \mathrm{m}$ for silicate dust in space rather than the $k \sim 10^{-8}$ of synthetic silicates because of impurities. Thus, the differences in sample composition could be responsible-our forsterite sample is synthetic and more pure than their natural sample. To check whether the 3 orders of magnitude difference between datasets is due to a difference in composition, we compared both datasets to $k$ that we derived from transmission spectra of gemstone olivine (source: Spat, Pakistan) with $10 \%$ Fe content (Fo90) and to a meteoritic sample (Brahin pallasite: Fo90). The San Carlos $k$ are still higher than the Fo90 and the pallasite by a similar amount to the synthetic forsterite. Thus, the difference in $k$ is not entirely due to composition but rather to the different thicknesses of the samples and to the quality of the sample (e.g., internal imperfections, degree of polish on the surface). Zeidler et al. (2011) used thin samples to measure $k$ in the regions of low absorption; the photomicrographs of the Sri Lanka sample in that work show visible cracks and imperfections in surface polish. Assuming that similar cracks are present in the thin samples of San Carlos olivine, this could cause scattering and thus artificially increase the $k$ values. The low values for $k$ seen for the forsterite in our work and for synthetic substances in general result in part from sample purity, but also from high quality polish and large sample size. For regions where $k$ is low, several mm-thick samples that are polished to a surface roughness equivalent to that of the spectrometer's reference mirror are required to constrain $k$. Absorbance in the NIR (especially from $5000-3000 \mathrm{~cm}^{-1}$, i.e., $2.0-2.857 \mu \mathrm{m}$ ) is very close to zero for any olivine that has no hydration and therefore cannot be measured in the lab to the degree of certainty that can be obtained in other ranges. Regardless of the exact values, all spectra in Fig. 2 indicate that laboratory-derived $k$ is substantially lower than constructed $k$ in the $0.2-5 \mu \mathrm{m}$ range.

\subsection{Theoretical modeling}

To determine what order of magnitude effect a user's choice in $n, k$ dataset will have on radiative transfer models, we performed a set of simple models on absorption crosssections, total flux and spectral energy distribution.

3.3.1 Absorption cross-section $\left(\left\langle\boldsymbol{C}_{\mathrm{abs}}\right\rangle\right)$ First, we calculated the average absorption cross-section, $\left\langle C_{\mathrm{abs}}\right\rangle$ (Fig. 3), of several laboratory and synthetic forsterite and olivine $n, k$ datasets used to represent forsterite for a continuous distribution of ellipsoids (CDE), using equations by Min et al. (2003). In the IR, $n$ and $k$ derived from the laboratory datasets produce equivocal $\left\langle C_{\mathrm{abs}}\right\rangle$. Longward of $8 \mu \mathrm{m}$ and into the far-IR, the $n$ and $k$ values derived from IR laboratory work (e.g., Fabian et al., 2001; Sogawa et al., 2006; Suto et al., 2006) differ slightly in resolution and $\mathrm{S} / \mathrm{N}$ but are effectively interchangeable for modeling. Compared to laboratory values, "astronomical silicate" $n, k$ from Draine (2003b) produce $\left\langle C_{\mathrm{abs}}\right\rangle$ values that are much higher in the VIS-NIR. Agreement between the Draine (2003b) "astronomical silicate" and our independently-derived laboratory values for forsterite is quite good shortward of $0.19 \mu \mathrm{m}$. Laboratory data from Huffman and Stapp (1973), which are part of the "astronomical silicate" $n, k$ (Draine, 2003b), produce values that dip slightly lower than ours between 0.2 and $0.3 \mu \mathrm{m}$ but are not as low as the values determined by Zeidler et al. (2011). From approximately $0.35-5 \mu \mathrm{m}$, the user's choice of $n, k$ dataset has the most effect on $\left\langle C_{\text {abs }}\right\rangle$. Within $0.3-2.5 \mu \mathrm{m}$, the user's choice of $k$ could vary $\left\langle C_{\mathrm{abs}}\right\rangle$ by up to 6 orders of magnitude. Longward of this range out to $5 \mu \mathrm{m}$, the choice of $k$ is still important, with an estimated "worst-case scenario" of two to three orders of magnitude difference.

3.3.2 DUSTY We used the 1-D radiative transfer program DUSTY (Nenkova et al., 2000), to demonstrate the effect of a user's choice of precise optical functions on the calculated spectral energy distribution (SED; Fig. 4). We aimed to test $n, k$ datasets that are made of all laboratory vs. all constructed vs. a combination of laboratory + constructed values, and selected three representative sets of optical functions for these cases, respectively: the $\mathrm{Mg}_{2} \mathrm{SiO}_{4}$ $n, k$ derived in this work, $n, k$ for "astronomical silicate" by Draine (2003b), and the $n, k$ data used by Wright $e t$ al. (2011) to approximate forsterite (a combination of Draine, $2003 b$ + IR laboratory values from Sogawa et al., 2006). In all cases, the models assume that dust forms and persists at a temperature of $1200 \mathrm{~K}$, which is reasonable for crystalline silicates (cf. Harker and Desch, 2002; Gail, 2004). The grains are assumed to have an MRN size distribution, i.e., $n(a)$ proportional to $a^{-q}$, where $n$ is the number of the grains in the size interval $(a, a+d a)$ and $q=3.5$; $a_{\min }=0.005 \mu \mathrm{m}$; and $a_{\max }=0.25 \mu \mathrm{m}$ (Mathis et al., 1977). Speck et al. (2009) demonstrated that for many reasonable grain-size-distributions the output SED is essentially unaffected. The inner edge of the dust shell is de- 


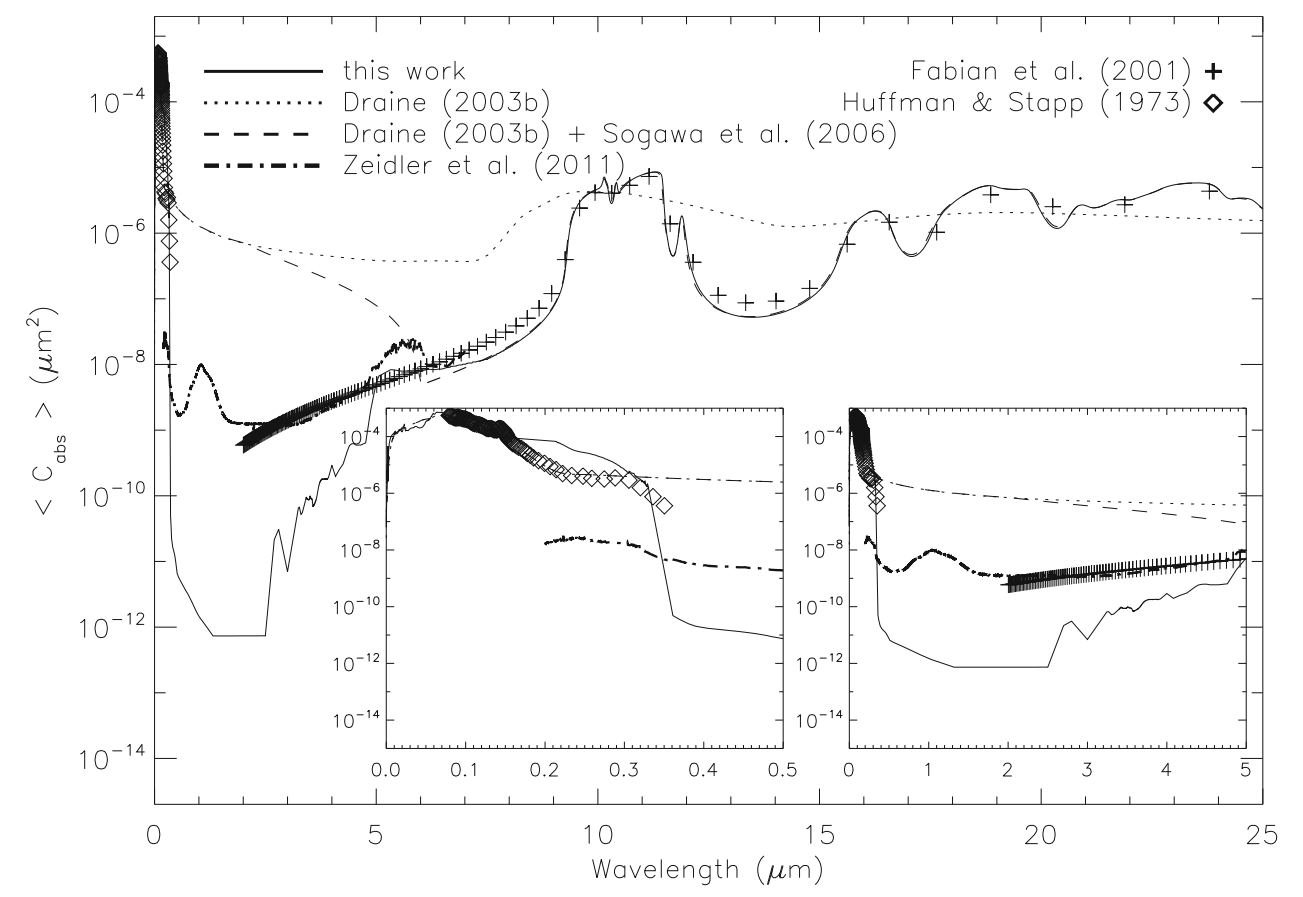

Fig. 3. Average absorption cross-section $\left\langle C_{\mathrm{abs}}\right\rangle$ for forsterite and olivine as a function of wavelength for a continuous distribution of ellipsoids with a particle radius $=0.01 \mu \mathrm{m}$, after Min et al. (2003). $\left\langle C_{\text {abs }}\right\rangle$ for a representative of modeler-constructed $n, k$ datasets ("astronomical silicate" from Draine, 2003b) are plotted against $\left\langle C_{\mathrm{abs}}\right\rangle$ laboratory $n, k$ from this work, (Draine (2003b) + Sogawa et al. (2006)) used in Wright et al. (2011), and various laboratory $n, k$ from Poteet et al. (2011). Every 30th data point is plotted for Fabian et al. (2001). $n, k$ datasets in the IR produce nearly identical $\left\langle C_{\text {abs }}\right\rangle$ values, whereas there is strong disagreement in results among the available UV-VIS-NIR $n, k$.

fined by the dust formation temperature; the outer edge is defined as 1000 times the inner radius. The density distribution of the dust grains is assumed to follow a $\mathrm{R}^{-2}$ distribution. Meanwhile the optical depth $\tau$ was set at $10 \mu \mathrm{m}$ to be 0.01 or 0.1 in order to test density effects. Two central star temperatures were used: $T_{\text {star }}=3000 \mathrm{~K}$ (typical for an asymptotic giant branch, or AGB, star) and 30,000 K (which is hot enough to have UV photons and ionize hydrogen). Speck et al. (2000) and DePew et al. (2006) showed that changing the star temperature by $\pm 1000 \mathrm{~K}$ did not significantly change the radiative transfer model's spectra (cf., Volk et al., 2000). While DUSTY can include other grain shapes, for simplicity of illustration and to minimize degeneracy between models, the dust grains in Fig. 4 are assumed to be spherical.

In Fig. 4 , the vertical axis plots $F_{\text {tot }}$, or total flux from the central star and surrounding dust (n.b., in optically thick cases, the star is not seen directly). The effect of modeling with different optical constants is clear for all models. For the low temperature AGB star $(T=3000 \mathrm{~K}$; Fig. 4(a, b)), the effect in the near-IR is not obvious. However, the lower opacity of the dust grains in the visible region for the new laboratory data gives rise to a faster drop-off in dust temperature, and thus the mid-IR dust features are much weaker. For the higher temperature $(T=30,000 \mathrm{~K})$ star, whereas the star emits most of its light in the UV, the changes due to choice of optical constants manifest in the near-IR, with the most extreme differences in $F_{\text {tot }}$ occurring shortward of $\lambda=5 \mu \mathrm{m}$ (Fig. 4(c, d) insets).

\section{Discussion}

\subsection{Implications for astronomers}

On whether to use constructed vs. laboratory data or a combination of the two to model stars, we have a few recommendations for astronomers. The DUSTY models in Fig. 4 show how model outputs are affected by the user's choice of $n$ and $k$, for different stellar temperatures and optical depths of the dust shell. The effect of $n, k$ on gross SED shape are less noticeable for the low temperature AGB star $(T=3000 \mathrm{~K}$; Fig. 4(a, b)), and thus, for modeling the overall shape of the SED, either constructed $n, k$ values like the "astronomical silicate" by Draine (2003b) or $n, k$ containing mostly laboratory data should have no adverse effect on models. However, there will be problems if one wants to look at the details of the dust emission features or the radial temperature distribution using the constructed $n, k$ values. For hotter stars (e.g., PN central stars) or any environment with an HII region, the absorption properties in the NIR, VIS, and (especially) UV become important. In these cases, the problem of using the constructed $n, k$ vs. $n, k$ derived from new laboratory data may not be obvious in the mid-IR emission bands, but is very clear in the VIS-NIR. DUSTY models do not include photoionization effects and these will undoubtedly be strongly affected by the change in UV-NIR flux that results from choosing the wrong optical constants. For any environment with significant UV flux, astronomers should use recent UV-VIS-NIR laboratory data rather than splicing in constructed $n, k$. Constructed $n, k$ are valuable at the shortest wavelengths because reasonably priced laboratory detectors typically cut off shortward of $0.19 \mu \mathrm{m}$. In the IR, laboratory $n, k$ are in consensus and offer an advantage for modeling crystalline material. 

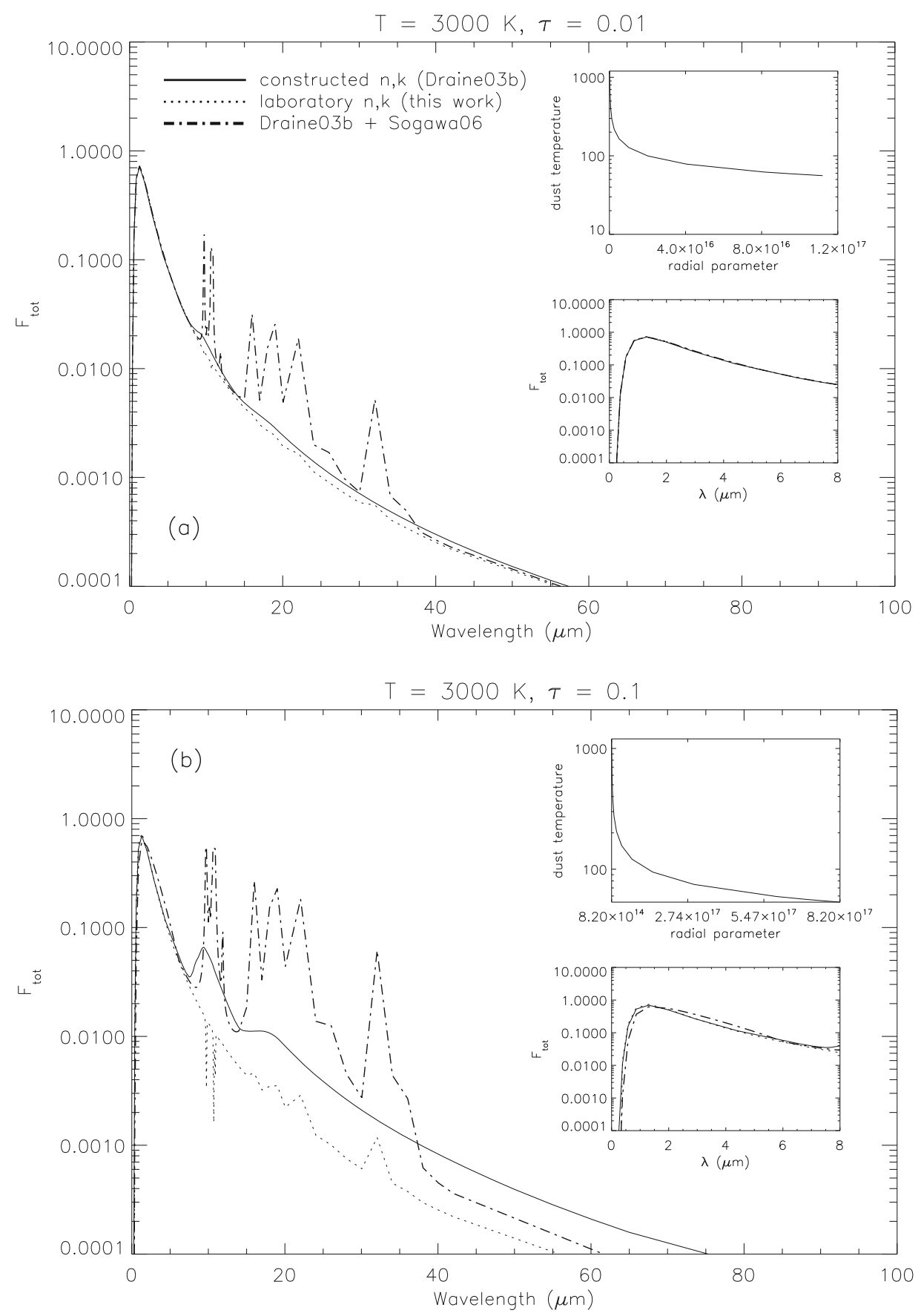

Fig. 4. DUSTY model runs for a spherical shell of dust around a central star of $T_{\mathrm{star}}=3000$ or $30,000 \mathrm{~K}$, and optical depth $\tau_{10 \mu \mathrm{m}}=0.01$ or 0.1 , using different options for silicate dust. Insets show behavior of total flux at shorter wavelengths and the dust temperature as a function of radial parameter. The choice whether to use all constructed $n, k$ ("astronomical silicate" from Draine, 2003b), all laboratory $n, k$ (forsterite, this work), or a combination of constructed and laboratory $n, k$ ("astronomical silicate" by Draine, 2003b + forsterite IR by Sogawa et al., 2006, merged by N. Wright) makes a profound difference to modeled $F_{\text {tot }}$.

There is also published precedent in astronomy journals to merge $n, k$ datasets for compounds made up of similar elements but having slight variances in composition or different structures. This often happens with forsterite, such that astronomical "forsterite" either refers to the $\mathrm{Mg}$ endmember $\mathrm{Mg}_{2} \mathrm{SiO}_{4}$ (e.g., Fig. 1 of this work; Sogawa et al., 2006; Suto et al., 2006), or to olivine with varying $\mathrm{Fe}$ content (Huffman and Stapp, 1973; Fabian et al., 2001; Zeidler et al., 2011; Fo90 and Brahin pallasite in Fig. 2 of this work). In the visible and shortward, spectral features are specific to cation and site, rather than structure. Figures 23 indicate that as one approaches the UV-vis, the Fe content dominates the spectral behavior, and it becomes essential to distinguish between pure forsterite from even $\mathrm{Mg}$-rich olivine. Apart from the difference in Fe content, forsterite, which is a crystalline compound, is very different from the blend of crystalline and disordered silicates that the Draine (2003b) data were derived from; see Speck et al. (2011) for 

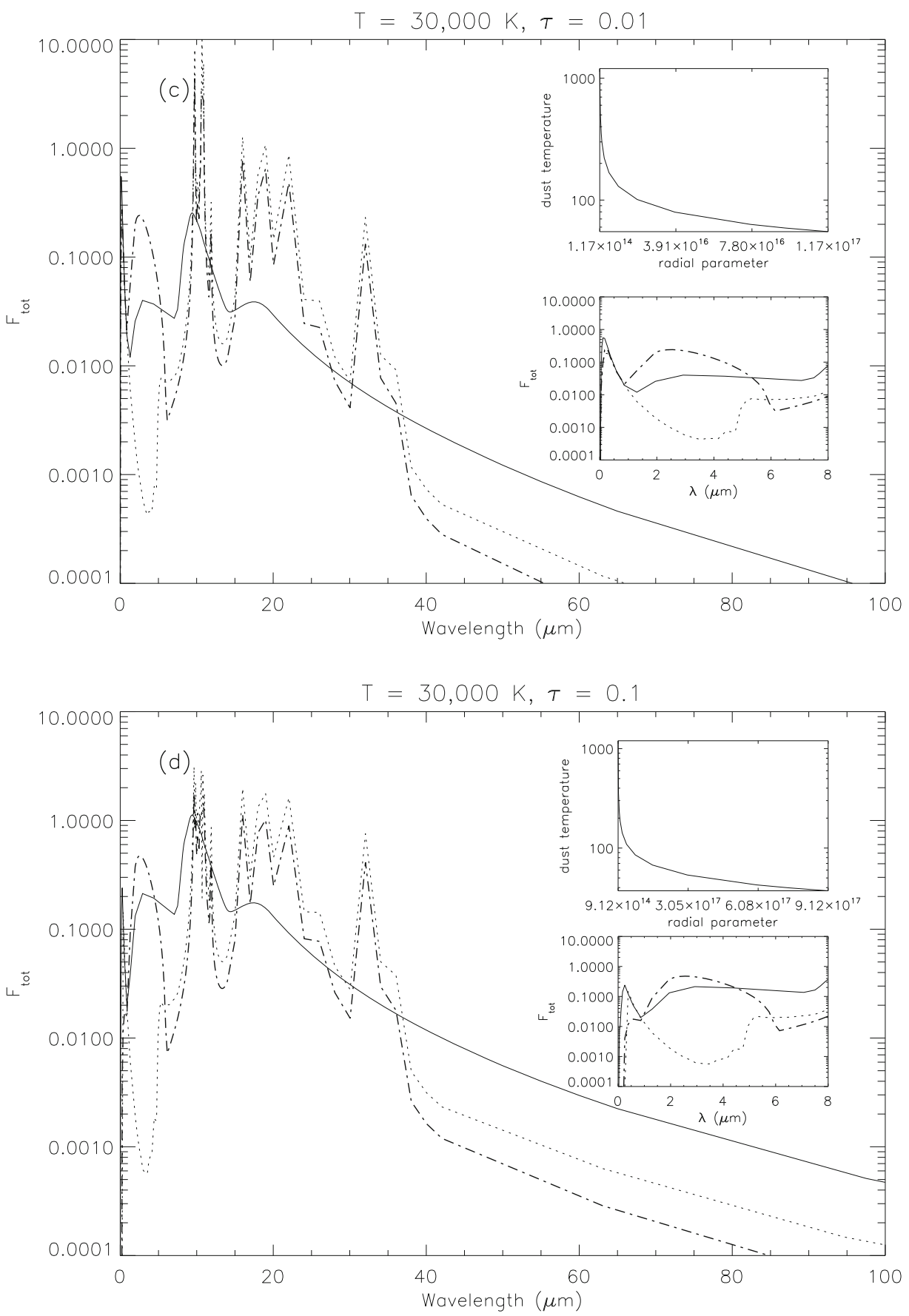

Fig. 4. (continued).

a recent discussion of the structural and spectral differences between crystalline, amorphous, and disordered silicates. The bulk composition of stardust lies between pyroxene $\left(\mathrm{M}_{2} \mathrm{Si}_{2} \mathrm{O}_{6}\right)$ and olivine $\left(\mathrm{M}_{2} \mathrm{SiO}_{4}\right)$, where $\mathrm{M}$ indicates metal cations, with $\mathrm{Mg}$ and Fe being the most abundant (Speck et al., 2008, 2011). Whereas most spectral observations of silicates in space have shown broad Si-O stretching features that are commonly attributed to amorphous solids, there are a handful of observations in which crystalline silicates have been clearly detected (e.g., Knacke et al., 1993; Crovisier et al., 1996; Waelkens et al., 1996; Waters et al., 1996; Spoon et al., 2006). Therefore, the best practice is to match both the chemical composition and structure (crystalline vs. disordered) even at the expense of maximizing wavelength coverage when merging $n, k$ values. Mixing related compounds ultimately creates confusion for identifications of pure forsterite vs. other types of silicate in space.

\section{Summary}

1) Forsterite is not interchangeable with Mg-rich olivine or disordered silicate. Any amount of Fe, even a trace, will affect the UV-vis substantially. Keeping the chemistry and structure consistent across all wavelengths and appropriate to the dust region of study is strongly 
recommended when comparing laboratory with astronomical data.

2) The choice whether to use modeler-constructed or laboratory optical functions, or to merge constructed data with lab data, makes a difference in calculated quantities (e.g., absorption cross-sections and total flux).

3) Laboratory $n, k$ data in the UV-VIS-NIR have been recently updated for forsterite and olivine. These values are much lower than for past "astronomical silicate" $n, k$ and should be used wherever possible.

4) Because it is very difficult to measure absorption coefficient and $k$ in the transparent region, there is not yet consensus on the UV-VIS-NIR behavior of forsterite and $\mathrm{Mg}$-rich olivine. To determine values of $k$ in the transparent region, samples must be large and highly polished.

Acknowledgments. This material is based upon work supported by the National Science Foundation under Grant No. AST1009544. The authors thank Harald Mutschke and an anonymous reviewer for providing helpful comments that enhanced the presentation of this work, Jonas Goldsand for laboratory assistance, and Nick Wright for copies of his forsterite $n, k$ tables. Electronic tables of the spectra shown in this work are available at http://galena.wustl.edu/ dustspec/idals.html. This is PSI Contribution No. 516 .

\section{References}

Colangeli, L., V. Mennella, A. Rotundi, P. Palumbo, and E. Bussoletti, Simulation of the cometary $10 \mu \mathrm{m}$ band by means of laboratory results on silicatic grains, Astron. Astrophys., 293, 927, 1995.

Crovisier, J., T. Y. Brooke, M. S. Hanner, H. U. Keller, P. L. Lamy, B. Altieri, D. Bockelee-Morvan, L. Jorda, K. Leech, and E. Lellouch, The infrared spectrum of comet C/1995 O1 (Hale-Bopp) at 4.6 AU from the sun, Astron. Astrophys., 315, L385, 1996.

David, P. and B. Pegourie, The complex dielectric function for circumstellar silicate grains based on the IRAS data, Astron. Astrophys., 293, 833, 1995.

Deer, W. A., R. A. Howie, and J. Zussman, An Introduction to the Rockforming Minerals, 2nd ed., 712 pp., Pearson Education Limited, Essex, 1992.

DePew, K., A. Speck, and C. Dijkstra, Astromineralogy of the $13 \mu \mathrm{m}$ feature in the spectra of oxygen-rich asymptotic giant branch stars. I. Corundum and spinel, Astrophys. J., 640(2), 971-981, 2006.

Dorschner, J., B. Begemann, Th. Henning, C. Jäger, and H. Mutschke, Steps toward interstellar silicate mineralogy. II. Study of $\mathrm{Mg}$-Fe-silicate glasses of variable composition, Astron. Astrophys., 300, 503, 1995.

Downs, R. T., The RRUFF project: an integrated study of the chemistry, crystallography, Raman and infrared spectroscopy of minerals, Program and Abstracts of the 19th General Meeting of the International Mineralogical Association in Kobe, Japan. O03-13, 2006.

Draine, B. T., Scattering by interstellar dust grains. I. Optical and ultraviolet, Astrophys. J., 598(2), 1017-1025, 2003a.

Draine, B. T., Scattering by interstellar dust grains. II. X-rays, Astrophys. $J .$, 598(2), 1026-1037, 2003b.

Ercolano, B., M. J. Barlow, P. J. Storey, and X.-W. Liu, MOCASSIN: a fully three-dimensional Monte Carlo photoionization code, Mon. Not. R. Astron. Soc., 340(4), 1136-1152, 2003.

Ercolano, B., M. J. Barlow, and P. J. Storey, The dusty MOCASSIN: fully self-consistent 3D photoionization and dust radiative transfer models, Mon. Not. R. Astron. Soc., 362(3), 1038-1046, 2005.

Fabian, D., T. Henning, C. Jäger, H. Mutschke, J. Dorschner, and O. Wehrhan, Steps toward interstellar silicate mineralogy. VI. Dependence of crystalline olivine IR spectra on iron content and particle shape, Astron. Astrophys., 378, 228-238, 2001.

Fournelle, I., Notes on some crystals of San Carlos olivine and EPMA standards, American Geophysical Union Fall Meeting, abstract \#V31E2009, 2009.

Gail, H.-P., Radial mixing in protoplanetary accretion disks. IV. Metamorphosis of the silicate dust complex, Astron. Astrophys., 413, 571-591,
2004.

Harker, D. E. and S. J. Desch, Annealing of silicate dust by nebular shocks at $10 \mathrm{AU}$, Astrophys. J., 565(2), L109-L112, 2002.

Henning, Th., V. B. Il'In, N. A. Krivova, B. Michel, and N. V. Voshchinnikov, WWW database of optical constants for astronomy, Astron. Astrophys. Suppl., 136, 405, 1999.

Hofmeister, A. M., K. M. Pitman, A. F. Goncharov, and A. K. Speck, Optical constants of silicon carbide for astrophysical applications. II. Extending optical functions from infrared to ultraviolet using singlecrystal absorption spectra, Astrophys. J., 696(2), 1502-1516, 2009.

Huffman, D. R. and J. L. Stapp, Optical measurements on solids of possible interstellar importance, 297-301, in Interstellar Dust and Related Topics (IAU Symp. 52), edited by J. M. Greenberg and H. C. van de Hulst, International Astronomical Union, Dordrecht, Boston, Reidel, 1973.

Jäger, C., H. Mutschke, B. Begemann, J. Dorschner, and Th. Henning, Steps toward interstellar silicate mineralogy. 1: Laboratory results of a silicate glass of mean cosmic composition, Astron. Astrophys., 292, 641, 1994.

Knacke, R. F., S. B. Fajardo-Acosta, C. M. Telesco, J. A. Hackwell, D. K. Lynch, and R. W. Russell, The silicates in the disk of beta Pictoris, Astrophys. J., 418, 440, 1993.

Koike, C., H. Mutschke, H. Suto, T. Naoi, H. Chihara, Th. Henning, C. Jäger, A. Tsuchiyama, J. Dorschner, and H. Okuda, Temperature effects on the mid-and far-infrared spectra of olivine particles, Astron. Astrophys., 449, 583, 2006.

Mathis, J. S., W. Rumpl, and K. H. Nordsieck, The size distribution of interstellar grains, Astrophys. J., 217, 425-433, 1977.

Miller, G. H., G. R. Rossman, and G. E. Harlow, The natural occurrence of hydroxide in olivine, Phys. Chem. Miner., 14, 461-472, 1987.

Min, M., J. W. Hovenier, and A. de Koter, Shape effects in scattering and absorption by randomly oriented particles small compared to the wavelength, Astron. Astrophys., 404, 35-46, 2003.

Nenkova, M., Z. Ivezic, and M. Elitzur, DUSTY: a publicly available code for modeling dust emission, in Thermal Emission Spectroscopy and Analysis of Dust, Disks, and Regoliths, edited by M. L. Sitko, A. L. Sprague, and D. K. Lynch, ASP Conf. Ser., 196, 77-82, 2000.

Ossenkopf, V., Th. Henning, and J. S. Mathis, Constraints on cosmic silicates, Astron. Astrophys., 261, 567, 1992.

Palik, E. D., ed. Handbook of Optical Constants of Solids, 999 pp., Academic Press, New York, 1998.

Pitman, K. M., A. M. Hofmeister, A. B. Corman, and A. K. Speck, Optical properties of silicon carbide for astrophysical applications. I. New laboratory infrared reflectance spectra and optical constants, Astron. Astrophys., 483(2), 661-672, 2008.

Pitman, K. M., C. Dijkstra, A. M. Hofmeister, and A. K. Speck, Infrared laboratory absorbance spectra of olivine: using classical dispersion analysis to extract peak parameters, Mon. Not. R. Astron. Soc., 406, 460, 2010.

Pollack, J. B., D. Hollenbach, S. Beckwith, D. P. Simonelli, T. Roush, and W. Fong, Composition and radiative properties of grains in molecular clouds and accretion disks, Astrophys. J., 421, 615, 1994.

Poteet, C. A., S. T. Megeath, D. M. Watson, N. Calvet, I. S. Remming, M. K. McClure, B. A. Sargent, W. J. Fischer, E. Furlan, L. E. Allen, J. E. Bjorkman, L. Hartmann, J. Muzerolle, J. J. Tobin, and B. Ali, A Spitzer infrared spectrograph detection of crystalline silicates in a protostellar envelope, Astrophys. J. Lett., 733(2), L2, doi:10.1088/20418205/733/2/L32, 2011.

Reynard, B., Single-crystal infrared reflectivity of pure $\mathrm{Mg}_{2} \mathrm{SiO}_{2}$ forsterite and $\left(\mathrm{Mg}_{0.86}, \mathrm{Fe}_{0.14}\right)_{2} \mathrm{SiO}_{4}$ olivine, Phys. Chem. Miner., 18, 19, 1991.

Rossman, G. R., Studies of $\mathrm{OH}$ in nominally anhyrous minerals, Phys. Chem. Miner., 23, 299-304, 1996.

Shankland, T. J., Synthesis and Optical Properties of Forsterite, Harvard University/Defense Technical Information Center, 170 pp., 1967.

Shankland, T. J. and K. Hemmenway, Synthesis of forsterite crystals, Am. Mineral., 48(1-2), 200, 1963.

Sogawa, H., C. Koike, H. Chihara, H. Suto, S. Tachibana, A. Tsuchiyama, and T. Kozasa, Astron. Astrophys., 451(1), 357-361, 2006.

Speck, A. K., M. J. Barlow, R. J. Sylvester, and A. M. Hofmeister, Dust features in the $10-\mu \mathrm{m}$ infrared spectra of oxygen-rich evolved stars, Astron. Astrophys. Suppl., 146, 437-464, 2000.

Speck, A. K., A. G. Whittington, and J. B. Tartar, The cosmic crystallinity conundrum: clues from IRAS 17495-2534, Astrophys. J., 687, L91, 2008.

Speck, A. K., A. B. Corman, K. Wakeman, C. H. Wheeler, and G. Thompson, Silicon carbide absorption features: dust formation in the outflows of extreme carbon stars, Astrophys. J., 691(2), 1202-1221, 2009. 
Speck, A. K., A. G. Whittington, and A. M. Hofmeister, Disordered silicates in space: a study of laboratory spectra of "amorphous" silicates, Astrophys. J., 740(2), 93, doi:10.1088/0004-637X/740/2/93, 2011.

Spoon, H. W. W., A. G. G. M. Tielens, L. Armus, G. C. Sloan, B. Sargent, J. Cami, V. Charmandaris, J. R. Houck, and B. T. Soifer, The detection of crystalline silicates in ultraluminous infrared galaxies, Astrophys. J., 638, 759, 2006.

Suto, H., H. Sogawa, S. Tachibana, C. Koike, H. Karoji, A. Tsuchiyama, H. Chihara, K. Mizutani, J. Akedo, K. Ogiso, T. Fukui, and S. Ohara, Mon. Not. R. Astron. Soc., 370(4), 1599-1606, 2006.

Tropf, W. J. and M. E. Thomas, Aluminum oxide $\left(\mathrm{Al}_{2} \mathrm{O}_{3}\right)$ revisited, in Handbook of Optical Constants of Solids, Vol. III, edited by E. D. Palik, pp. 653-682, 999 pp., Academic Press, New York, 1998.

Volk, K., G.-Z. Xiong, and S. Kwok, Infrared Space Observatory spectroscopy of extreme carbon stars, Astrophys. J., 530(1), 408-417, 2000.

Waelkens, C. et al., SWS observations of young main-sequence stars with dusty circumstellar disks, Astron. Astrophys., 315, L245, 1996.

Waters, L. B. F. M. et al., Mineralogy of oxygen-rich dust shells, Astron. Astrophys., 315, L361, 1996.
Weeks, R. A., J. C. Pigg, and C. B. Finch, Charge-transfer spectra of $\mathrm{Fe}^{3+}$ and $\mathrm{Mn}^{2+}$ in synthetic forsterite $\left(\mathrm{Mg}_{2} \mathrm{SiO}_{4}\right)$, Am. Mineral., 59, 1259, 1974.

Wright, N. J., M. J. Barlow, B. Ercolano, and T. Rauch, A 3D photoionization model of the extreme planetary nebula NGC 6302, Mon. Not. R. Astron. Soc., 418, 370-389, doi:10.1111/j.1365-2966.2011.19490.x, 2011.

Yang, X.-Z. and H. Keppler, In-situ infrared spectra of $\mathrm{OH}$ in olivine to $1100^{\circ} \mathrm{C}$, Am. Mineral., 96(2-3), 451-454, doi:10.2138/am.2011.3717, 2011.

Zeidler, S., T. Posch, H. Mutschke, H. Richter, and O. Wehrhan, Near-infrared absorption properties of oxygen-rich stardust analogs. The influence of coloring metal ions, Astron. Astrophys., 526, A68, doi:10.1051/0004-6361/201015219, 2011.

K. M. Pitman (e-mail: pitman@psi.edu), A. M. Hofmeister, and A. K. Speck 